\title{
Endodontic Management of a Three-rooted Mandibular First Premolar associated with Taurodontism
}

\author{
${ }^{1}$ Swati Ishwar Wadekar, ${ }^{2}$ Dipali Yogesh Shah, ${ }^{3}$ Jimish Rajiv Shah, ${ }^{4}$ Kinjal Mahesh Gathani
}

\begin{abstract}
Introduction: Taurodontism is a morpho-anatomic variation in tooth anatomy that rarely affects mandibular premolars. Literature is replete with mandibular first premolars presenting atypical additional root canals, but concurrent presence of three roots and taurodontism is exceptional.
\end{abstract}

Materials and methods: This case report describes the successful endodontic diagnosis and management of a rare case of taurodontism in a mandibular first premolar with chronic apical periodontitis. Cone beam computed tomography (CBCT) facilitated the diagnosis of three roots and root canals associated with concurrent taurodontism. The taurodont premolar required exceptional and diligent care in each segment of endodontic treatment for the complex root canal system.

Results: Taurodontism-affected premolars present with challenges in endodontic therapy as they have apically displaced pulp chambers with limited width. Cone beam computed tomography and dental operating microscope can facilitate evaluation for teeth with such complex anatomy and their successful endodontic management.

Keywords: Anatomic variations, Cone beam computed tomography, Endodontic therapy, Mandibular first premolar, Taurodontism.

How to cite this article: Wadekar SI, Shah DY, Shah JR, Gathani KM. Endodontic Management of a Three-rooted Mandibular First Premolar associated with Taurodontism. J Oper Dent Endod 2016;1(2):90-92.

Source of support: Nil

Conflict of interest: None

\section{INTRODUCTION}

The success of endodontic therapy depends on the thorough knowledge of the internal morphology of root canal system. Failure to recognize variations in root and root canal anatomy may result in unsuccessful endododontic treatment. ${ }^{1}$ Of all the teeth treated with

\footnotetext{
1,3,4 Postgraduate Student ${ }^{2}$ Professor

${ }^{1-4}$ Department of Conservative Dentistry and Endodontics Sinhgad Dental College and Hospital, Pune, Maharashtra, India

Corresponding Author: Dipali Yogesh Shah, Professor Department of Conservative Dentistry and Endodontics Sinhgad Dental College and Hospital, Pune, Maharashtra, India Phone: +912024351303, e-mail: dipali.nagda@yahoo.com
}

primary endodontic therapy, the mandibular first premolars have the highest failure rate. This accreditation is primarily because of their variable and complex root canal morphology. ${ }^{2}$ Normally, mandibular first premolars present with a single root and a single root canal system. The incidence of two roots is $1.8 \%$, while that of three roots is only $0.2 \%$ in these teeth. ${ }^{3}$

Taurodontism refers to an atypical morpho-anatomical variation in teeth that exhibit three characteristic features, namely: The floor of the pulp chamber is apically displaced, lack of the usual constriction at the cement-enamel junction of the teeth, and elongated pulp chamber. ${ }^{4}$ It chiefly affects the mandibular second molars but the mandibular premolars are rarely affected. The concurrent presence of three roots in a mandibular premolar and taurodontism is exceptional and its endodontic treatment would be undoubtedly challenging. This clinical case report describes the use of cone beam computed tomography (CBCT) in the successful endodontic management of a challenging case of a taurodont mandibular first premolar with three roots.

\section{CASE REPORT}

A29-year-old female patient reported to the department of Conservative Dentistry and Endodontics, Sinhgad Dental College and Hospital, Pune, with a chief complaint of spontaneous pain in the lower right posterior region of the jaw for past 3 days. The tooth had a history of prolonged sensitivity to hot and cold. On clinical examination, \#44 exhibited a deep occlusal carious lesion that was tender on percussion, with no mobility. Vitality testing of the involved tooth with warm gutta-percha caused an intense lingering pain, whereas electric pulp testing gave an early response. Intraoral periapical radiograph revealed an occlusal radiolucency approaching the pulp space with widening of the apical periodontal ligament space (Figs 1A to E). It also revealed presence of multiple root canal morphology. Radiographically, mid-root diameter appeared to be almost equal to the coronal diameter of the 44. Based on clinical and radiographic examinations, tooth \#44 was diagnosed with symptomatic irreversible pulpitis with apical periodontitis secondary to dental caries. A periapical radiograph of contralateral tooth \#34 showed 

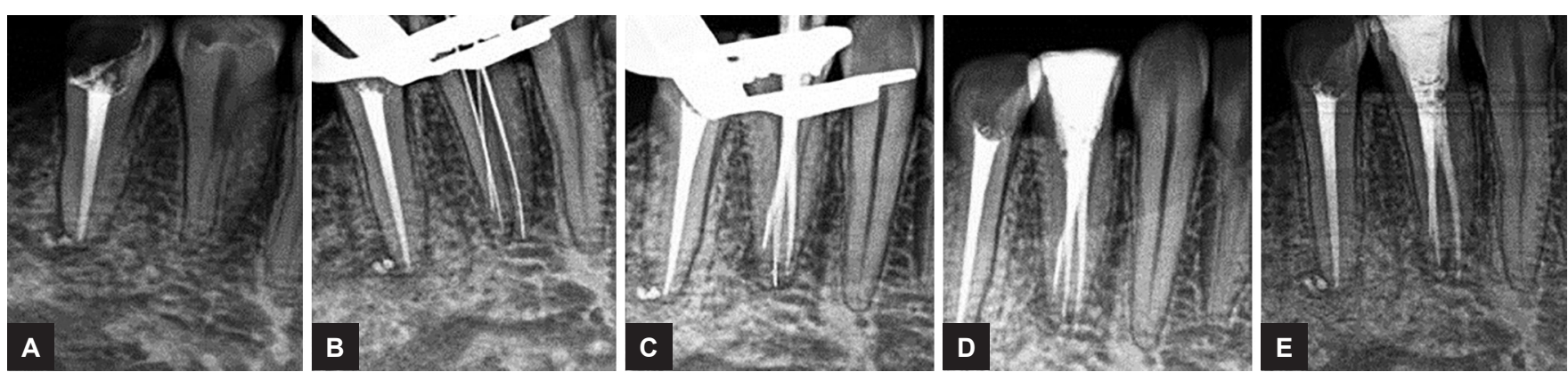

Figs 1A to E: (A) Preoperative intraoral diagnostic radiograph of tooth \#44; (B) working-length radiograph showing three root canals; (C) master cone selection radiograph of tooth \#44; (D) postobturation radiograph and coronal seal with composite restoration; and (E) six-month follow-up radiograph of tooth \#44
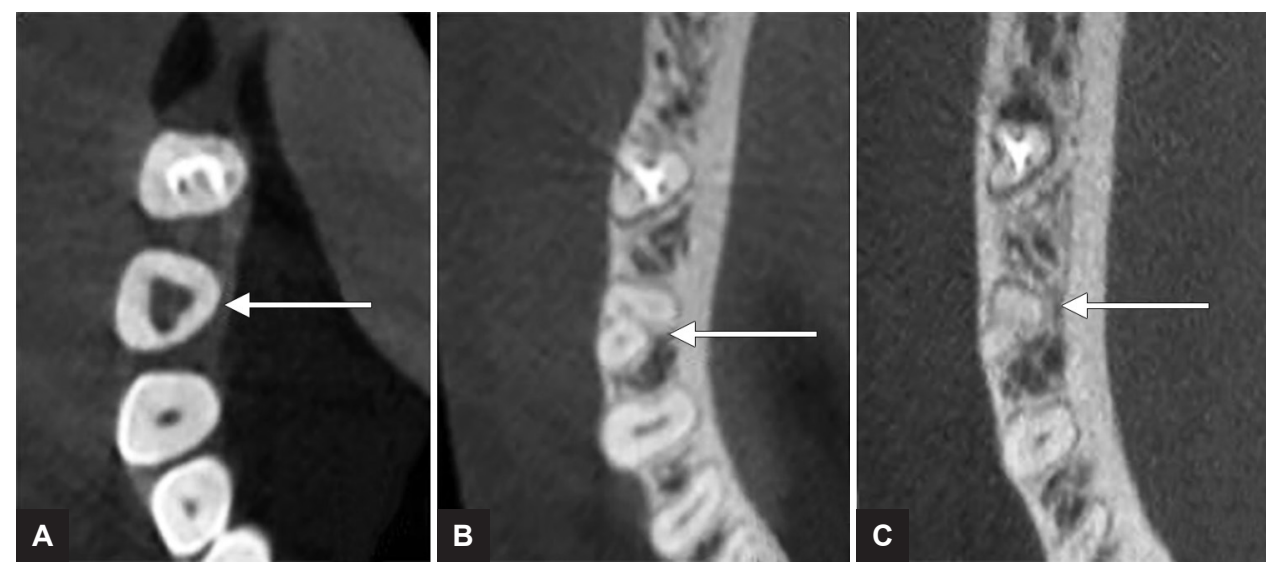

Figs 2A to C: Axial views CBCT images of tooth \#44 (A) At coronal one-third level of the root;

$(B)$ at middle one-third level of the root; and (C) at cervical one-third level of the root

presence of single root and root canal system and a similar anomaly on the contralateral tooth was ruled out. Cone beam computed tomography imaging of tooth \#44 was advised to further evaluate the unusual tooth morphology and nonsurgical endodontic treatment was recommended.

An informed consent was obtained from the patient, and multislice $\mathrm{CBCT}$ imaging of the mandible was performed with field of view (FOV) $40 \times 40 \mathrm{~mm}$ and a tube voltage $90 \mathrm{kV}$ and tube current $5 \mathrm{~mA}$ with an exposure time of 9.4 seconds (Kodak Dental Systems Carestream Health, NY, USA). The axial images of the \#44 confirmed the presence of three roots and three root canals separating at the apical third level of the root and exiting as three separate apical foramina. Images also confirmed the concurrent presence of taurodontism (Figs 2A to C). Later, Shiffmann and Channenel index was used to score the taurodont premolar.

Access was gained to the pulp chamber after administration of local anesthesia ( $2 \%$ lidocaine with 1:80,000 adrenaline) and rubber dam isolation. To gain sufficient straight-line access, the conventional opening was modified into one that was wider mesiodistally. Three canal orifices were identified under the dental operating microscope were mesiobuccal, distobuccal, and lingual. Canal patency was established with \#10 K-files (Dentsply Maillefer, Bal- laigues, Switzerland). Canal orifices were enlarged and the working length was determined using Root $Z X$ apex locator (J. Morita, Tokyo, Japan) and later confirmed radiographically (Fig. 1). The root canal system was cleaned and shaped using hand K-files. A\#20 K. file separated in the mesiobuccal canal and it was bypassed and instrumented till apical \#30. The other two canals were prepared using hand ProTaper files up to \#F2, using $2.5 \%$ sodium hypochlorite and $17 \%$ ethylenediamine tetraacetic acid (EDTA) as irrigants. An amount of 17\% EDTA was used for 1 minute as the final rinse, followed by saline. Subsequently, canals were obturated with F2 Protaper gutta-percha points (Dentsply Maillefer, Ballaigues, Switzerland) and AH plus root canal sealer (Dentsply Maillefer, Ballaigues, Switzerland), and the long pulp chamber was filled using a lateral condensation technique (Fig. 2). Composite resin was used for the postendodontic restoration. After 6 months, the tooth remained asymptomatic and clinically sound. A follow-up radiograph was taken and a porcelain fused to a metal crown was advised as the final restoration.

\section{DISCUSSION}

Tooth root morphology is chiefly determined genetically, but it may be modified environmentally. Taurodontism is chiefly associated with molars and rarely in mandibular 
premolars. ${ }^{5}$ It may be associated with various syndromes. ${ }^{4}$ In the present case, systemic disturbances or malformations could not be identified and hence, it was considered as nonsyndromic taurodontism. Also, inheritable etiology could not be determined because the patient's family members were unavailable for radiographic examination.

In the Indian population, Moayedi and Lata ${ }^{6}$ reported a case of mandibular premolar with three root canals and three apical foramina, but taurodontism was not confirmed. Nallapati ${ }^{7}$ reported three root canals in the mandibular first and second premolars in the same patient. The present case additionally had apically displaced pulp chamber leading to a taurodont premolar. Applying the criteria of Shiffmann and Channenel, ${ }^{8}$ an index score of $56 \%$ was obtained and the premolar was termed a hypertaurodont (40-75\%). The CBCT scanning images aided the operator in the estimation of orifice locations, canal anatomy, and to rule out any additional aberrations, thus overcoming limitations of periapical radiography. ${ }^{9}$

The close proximity of the root canal orifices in the limited width of the pulp chamber floor that were located deep in the middle third of the root trunk was the chief endodontic challenge. Hence, the use of the dental operating microscope was imperative to enhance the clinician's ability to locate and access the orifices. Rotary instrumentation was not used in the apically located root canals, rather hand instrumentation was preferred as taurodont roots with small width may increase the chances of dentinal crack formation and subsequent fractures. ${ }^{10}$ Despite all the precautions, instrument separation occurred in the mesiobuccal canal but it was successfully bypassed and endodontic healing was uneventful after obturation. Establishment of a coronal seal with postendodontic restoration and follow-up confirmed the endodontic healing of this three-rooted hypertaurodont premolar.

\section{CONCLUSION}

Though taurodontism is relatively uncommon in premolars, the dental practitioner should bear in mind the clinical significance of this condition. In this threerooted taurodontism case, use of high-power magnification aided in the localization of the canals and CBCT ruled out any additional aberrations in the root canal morphology.

\section{REFERENCES}

1. Hoen MM, Pink FE. Contemporary endodontic retreatments: an analysis based on clinical treatment findings. J Endod 2002 Dec;28(12):834-836.

2. Slowey RR. Root canal anatomy. Road map to successful endodontics. Dent Clin North Am 1979 Oct;23(4):555-573.

3. Cleghorn BM, Christie WH, Dong CC. The root and root canal morphology of the human mandibular first premolar: a literature review. J Endod 2007 May;33(5):509-516.

4. Jafarzadeh H, Azarpazhooh A, Mayhall JT. Taurodontism: a review of the condition and endodontic treatment challenges. Int Endod J 2008 May;41(5):375-388.

5. Llamas R Jimenez-Planas A. Taurodontism in premolars. Oral Surg Oral Med Oral Pathol 1993 Apr;75(4):501-505.

6. Moayedi S, Lata D. Mandibular first premolar with three canals. Endodontology 2004;16:26-29.

7. Nallapati S. Three canal mandibular first and second premolars: a treatment approach. J Endod 2005 Jun;31(6):474-476.

8. Shiffmann A, Channenel I. Prevalence of taurodontism found in radiographic dental examination of 1,200 young adult Israeli patients. Community Dent Oral Epidemiol 1978 Jul;6(4): 200-203.

9. Alamri HM, Sadrameli M, Alshalhoob MA, Sadrameli M, Alshehri MA. Applications of CBCT in dental practice: a review of the literature. Gen Dent 2012 Sep-Oct;60(5):390-400.

10. Prakash R, Vishnu C, Suma B, Velmurugan N, Kandaswamy D. Endodontic management of taurodontic teeth. Indian J Dent Res 2005 Oct-Dec;16(4):177-181. 